\title{
Creatividad en pista II: Jugando con las emociones
}

\author{
Lucía Jiménez
}

\section{RESUMEN}

En este artículo se propone como regular las emociones, y retomar el componente de disfrute por el cual todo jugador/a comenzó a jugar al tenis. Para ello se toma como base la visión cognitivo-conductual de las emociones y se proponen ejercicios aplicables a todos los niveles de práctica

\author{
Palabras clave: Emociones, \\ juego, perspectiva cognitivo- \\ conductual
}

Recibido: 30 Mayo 2018

Aceptado: 28 Junio 2018

Autor correspondiente:

Lucía Jiménez.

Correo electrónico:

lucia@feelinflow.com

\section{INTRODUCCIÓN}

"En vez de ser cosas de lujo, las emociones son una forma muy inteligente de motivar al organismo hacia ciertos resultados" (Antonio Damasio)

"Por primera vez en mi carrera profesional no he sido dueño de mis emociones en la pista, no he tenido el control de mis nervios, he pasado malos ratos, pero lo he superado" (Rafael Nadal).

Las emociones son reacciones psicofisiológicas ante acontecimientos ambientales que se presentan (Lazarus, 2000). Dentro de la psicología, la perspectiva cognitivo-conductual constituye uno de los principales enfoques desde el cual se han abordado las mismas. Desde esta visión y tal y como comentaría Epicteto siglos atrás, "No son las cosas las que atormentan a las personas, sino la opinión que de ellas tienen", es decir, no son las circunstancias externas las que determinan cómo nos sentimos, sino la interpretación que hacemos de los acontecimientos. En este sentido, ante una contrariedad como pueda ser perder un primer set, existirán muchas reacciones diferentes en función de los pensamientos que tenga el jugador, y eso determinará tanto la emoción, como lo que suceda a continuación.

La propuesta para este artículo consiste en trabajar la emoción en pista mediante la modificación consciente del pensamiento y la conducta, principales responsables de las emociones. Para ello se tendrá presente la noción de "juego" inherente al tenis, aplicable a todos los niveles de práctica.

\section{JUGANDO CON LAS EMOCIONES}

Cuesta pensar que hubo un niño/a alguna vez que, independientemente de su origen y circunstancias personales, no comenzase a jugar al tenis por pura diversión, no pasase horas y horas golpeando la pelota contra una pared o contra distintas "víctimas" (entrenadores/as, padres, compañeros...) con las que saciar las ganas y la ilusión por mejorar, por divertirse. Las emociones despertadas en esas circunstancias (alegría, felicidad, amor, sorpresa, enfado, miedo, etc.) supusieron el motor que permitió persistir más allá del cansancio o de la propia tensión del momento, y es que no podemos olvidar que todas las emociones, incluidas aquellas que etiquetamos como negativas, tienen una función adaptativa, una función social y una función motivacional (Reeve, 1994) que mueven nuestra energía hasta límites insospechados.

Dando por sentado que queremos ayudar a nuestros jugadores/as a despertar emociones que les permitan persistir en la mejora y en el esfuerzo, y que a su vez les sirvan como aprendizaje para otras circunstancias de su vida, ¿por qué no entonces remontarnos a esas sensaciones iniciales que impulsaron al jugador/a a continuar, allá por sus comienzos? Nos referimos fundamentalmente al disfrute. En la propia expresión de nuestro deporte está implícita la idea de juego, hablamos de jugar al tenis. Y no es casualidad. 


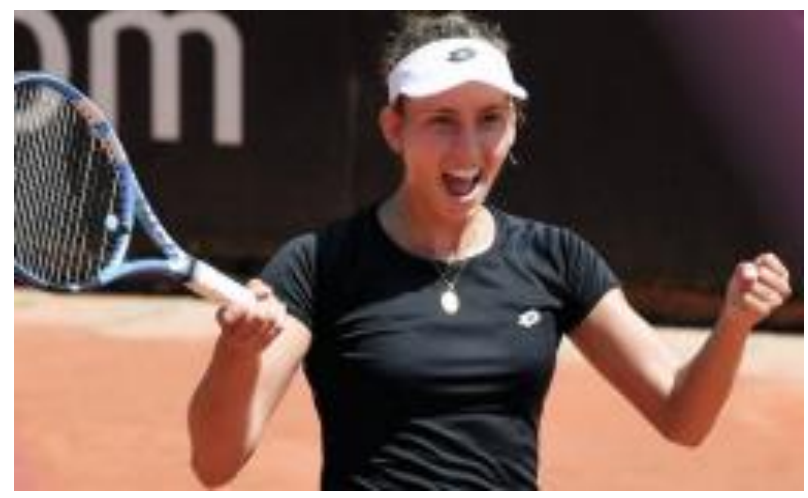

el ser humano, el niño/a por excelencia, aprende jugando, prueba de ello es la numerosa documentación que muestra la relación entre diversión y rendimiento óptimo en deporte (Jackson, 2000) y concretamente en tenis (e.g. Young, 2016).

"Cuando no disfrute del tenis, colgaré la raqueta" (David Ferrer).

Así pues, ¿en qué momento se olvida mantener la idea de juego presente? Si bien el tenis es un deporte en el que se profesionaliza tempranamente al jugador, existiendo mucho dinero, contratos y compromisos de por medio, lo que no varía -más allá del nivel de práctica- es la noción de disfrute.

$Y$ es que el dinero o la fama son un poderoso motor pero, si queremos jugadores/as que persistan en su práctica conviene fomentar el disfrute y las emociones agradables. Y cuidado, esto no significa que las emociones desagradables no deban estar presentes, deben estarlo en su justa medida. Cabe también decir que con disfrutar no nos referimos a estar siempre riendo, nos referimos a ser capaz de ver las circunstancias difíciles como oportunidades de crecimiento, como retos, y disfrutar de ello.

\section{Pautas para Jugar con las Emociones:}

No hay reglas fijas. Como entrenador/a, aprovecha para despertar tu creatividad en los ejercicios que propongas, te sorprenderá observar cómo a raíz de ello, tus jugadores/as también empezarán a ser más creativos:

1. Relativiza tus emociones, no las tomes tan en serio. Por definición, las emociones son respuestas psicofisiológicas cuya duración es de segundos o minutos (Oatley y Jenkins, 1996), por tanto pasarán, es cuestión de tiempo.

2. Normaliza su aparición. Las emociones son inherentes a nuestra naturaleza, siempre nos acompañarán. Empieza a pensar de acuerdo a esta idea y la intensidad de la emoción en momentos de tensión se reducirá considerablemente.

3. Toma ventaja de las mismas. Las emociones proporcionan mucha información sobre ti, aprovecha la ocasión para observar tus pensamientos y comportamientos más comunes.

4. Fomenta emociones positivas. Está demostrado que amplían los repertorios de pensamiento y acción (Fredrickson, 2013), además de relacionarse positivamente con la atención, la creatividad y la toma de decisiones (Fernández-Abascal, Jiménez Sánchez, Martín Díaz, \& Domínguez Sánchez, 2010).

5. Disfruta: Una de las principales separaciones entre tu estado actual y jugar a tu máximo nivel reside en la presencia de diversión (Loehr \& Fiske, 1995).

6. Visión panorámica: No todo es emocional. Haz una foto mental de tu situación, ¿tal vez necesites reforzar algún aspecto técnico o físico?

7. Líneas Rojas. Establece aquellas actitudes que bajo ningún concepto vas a traspasar.

8. Aliadas: Todas las emociones tienen un mensaje muy útil, incluidas las emociones desagradables. Si en lugar de frustrarnos por su aparición o enfadarnos aún más con nosotros, las utilizamos como aliadas, podremos revertir las consecuencias negativas y tomarlas como una oportunidad de mejora. Un ejemplo de ello es la ira, si bien constituye una de las emociones más perjudiciales y desgraciadamente más presentes dentro del circuito, también es cierto que moviliza gran cantidad de energía. ¿Cómo sería reorientar esa energía, en lugar de para golpear la raqueta, para luchar todas las bolas el resto del partido?

\section{Ejercicios:}

Desde la visión cognitivo-conductual existen numerosos ejercicios que nos permiten modificar o inducir determinadas emociones. A continuación presentamos algunos ejemplos:

1. Ponle cara a tus emociones: En general, cuesta trabajo nombrar la emoción por el simple hecho de que no estamos familiarizados con las mismas, tenemos un vocabulario emocional muy reducido que limita enormemente nuestra capacidad de expresión. Comencemos con un ejercicio muy básico y muy efectivo a su vez que consiste en nombrar la emoción. Para ello, nos ayudaremos de emoticonos, con los cuales los jugadores/as sí están familiarizados. Mediante ellos abriremos la puerta al estado emocional y eso dará pie a que podamos tener una conversación posterior más profunda.

2. Personajes: Cuando tu jugador/a se queda atrapado en la emoción, ¿a qué personaje se parece? ¿qué papel está representando? En primer lugar, establece junto a él/ella ese personaje, pregúntaselo. Tal vez sea un Diablo de Tasmania, que arrasa con todo independientemente del momento del partido; tal vez sea un Pájaro loco, el cual se limita a pegar 
golpes sin pensar; tal vez sea un Superman/Superwoman, que tiene que hacerlo todo perfecto en todo momento; tal vez sea un Mayordomo sumiso el cual camina con la cabeza agachada y pidiendo permiso; tal vez sea un Coche de Bomberos que sólo reacciona cuando la cosa se pone tensa; tal vez sea un Alma caritativa que antepone los intereses de los demás al suyo propio...

Hay miles de posibilidades, pero ojo, es importante que tu jugador/a esté implicado en la elección y que, en ningún caso, sea impuesto $u$ ofensivo, principalmente porque ese personaje le ayudó a evolucionar hasta donde ahora está, y por tanto merece agradecimiento. Se trata simplemente de tomar conciencia de que cuando se pone en ese estado, se deja llevar por unas emociones-pensamientos-acciones que le impiden dar lo mejor de sí.

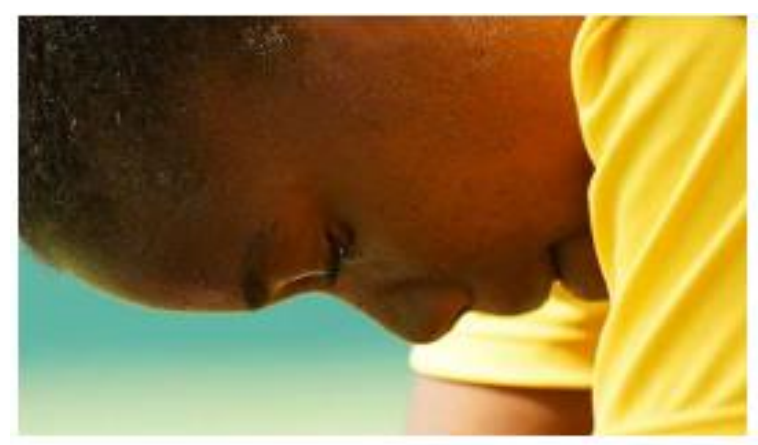

En segundo lugar, propón a tu jugador/a que exagere el personaje hasta el final. Llevando al "extremo permitido" el personaje en el que está atrapado emocionalmente podrá relativizar con mucha facilidad, y darse cuenta de lo "ridículo" que puede llegar a ser cuando se identifica durante mucho tiempo con él/ella.

Finalmente, y una vez dominadas las dos primeras fases con fluidez, estableced -nuevamente de manera conjunta- un personaje que le potencie, que le lleve a dar lo mejor de sí. Que lo detalle sobre papel, que describa sus pensamientos habituales, su forma de moverse, de vestirse, de caminar en la pista, sus gestos de ánimo, sus emociones... Y que trate de convertirse cada día un poquito en él/ella.

3. Mantra: ¿Cuál es ese mensaje, ese pensamiento o esa creencia que necesitas repetirte para dar lo mejor de ti, para esforzarte o simplemente para relajarte y poder disfrutar de tu tenis? Establece y escribe una o dos afirmaciones y repítelas en diferentes ocasiones: antes de entrenar, durante el entreno, y antes de dormir. Si lo deseas, puedes guardarlas en tu bolsillo o colgarlas en un lugar visible para ti. Hazlo durante tres semanas y después sustitúyelas por otras.

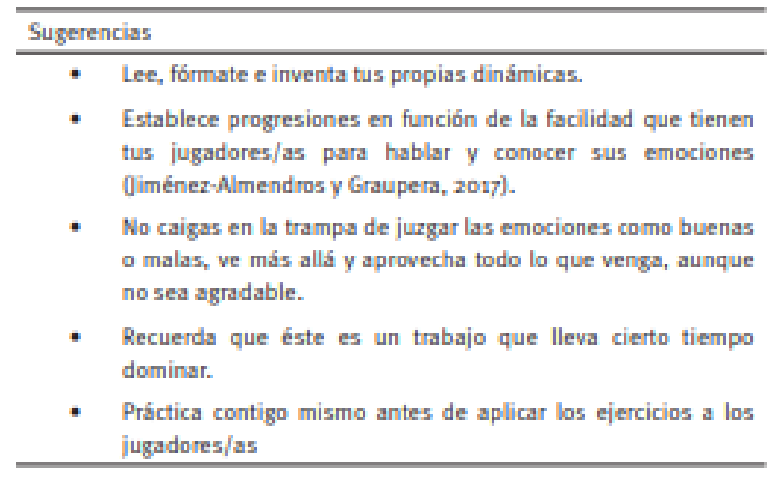

Tabla 1. Sugerencios para entrenadores

\section{CONCLUSIONES}

En los últimos años se ha puesto muy de moda hablar sobre las emociones, lo presentes que están durante un partido, cómo sería posible regularlas... A nuestro parecer, resulta especialmente importante empezar por lo más básico (identificarlas, nombrarlas, reconocerlas) para poder actuar consecuentemente. De las diversas formas que se pueden trabajar hemos propuesto unos ejercicios que abordan la emoción a nivel de conducta y pensamiento, considerándolos tan sencillos como eficaces.

Normalizar o cambiar el curso de una emoción durante un entrenamiento o partido da lugar a una experiencia totalmente diferente de la situación, con unos resultados completamente distintos también, mucho más satisfactorios, duraderos y constructivos. En tu mano está, como entrenador/a, poner a disposición de tus jugadores experiencias de aprendizaje y disfrute. Recuerda, tú eres su mejor ejemplo, si te ven disfrutar, es muy probable que ellos también disfruten.

\begin{tabular}{l}
\hline Claves \\
\hline - La emoción es pasajera. \\
* Fomenta emociones agradables sin menospreciar las \\
desagradables, conviértelas en tus aliadas. \\
- Juega con las emociones. Plantea retos a tus jugadores para \\
que disfruten mientras se superan. \\
\hline
\end{tabular}

Tablo 2. Cloves a recordar

\section{REFERENCIAS}

Fernández-Abascal, E. G., Jiménez Sánchez, M. P., Martín Díaz, M. D., \& Domínguez Sánchez, F. J. (2010). Psicología de la emoción. Madrid: Editorial Universitaria Ramón Areces

Fredrickson, B. L. (2013). Positive Emotions Broaden and Build. In P. Devine \& A. Plant (Eds.), Advances in Experimental Social Psychology (Vol. 47). Burlington: Academic Press. https://doi.org/10.1016/B978-0-12-407236-7.00001-2 
Jackson, S. A. (2000). Joy, fun, and flow state in sport. In Y. L. Hanin (Ed.), Emotions in sport (pp. 135-155). Champaign, IL: Human Kinetics https://doi.org/10.5040/9781492596233.ch-006

Jiménez-Almendros, L. y Graupera, J. L. (2017). Perfil de Afrontamiento Cognitivo-Emocional en tenistas de competición. E-Coach Revista Electrónica del Técnico de Tenis, 29 (9), 25-33.

Lazarus, R. S. (2000). How emotions influence performance in competitive sports. The Sport Psychologist, 14(3), 229-252. https://doi.org/10.1123/tsp.14.3.229

Loehr, J. y Fiske, S. (1995). 6 keys to getting \& staying in the zone. Tennis, 31 (3), 36.

Oatley, K. y Jenkins, J.M. (1996). Understanding emotions. Cambridge: MA: Blackwell Scientific.

Reeve, J. (1994). Motivación y Emoción. Madrid: McGraw-Hill.

Young, J. (2016). Enjoyment: Fresh clues for coaches. ITF Coaching and Sport Science Review, 68, 21-23. https://doi.org/10.52383/itfcoaching.v24i68.176
CONTENIDO ITF ACADEMY RECOMENDADO (HAZ CLICK ABAJO)

\section{ITF Academy}

Derechos de Autor (c) 2018 Lucía Jiménez

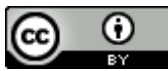

Este texto está protegido por una licencia CreativeCommons 4.0.

Usted es libre para Compartir —copiar y redistribuir el material en cualquier medio o formato-y Adaptar el documento - remezclar, transformar y crear a partir del material- para cualquier propósito, , incluso para fines comerciales, siempre que cumpla la condición de:

Atribución: Usted debe dar crédito a la obra original de manera adecuada, proporcionar un enlace a la licencia, e indicar si se han realizado cambios. Puede hacerlo en cualquier forma razonable, pero no de forma tal que sugiera que tiene el apoyo del licenciante o lo recibe por el uso que hace de la obra.

Resumendelicencia - Textocompletodelalicencia 